\title{
Étudier les usages des plateformes pédagogiques numériques : quelles passerelles entre STIC et SHS ?
}

Angeliki Koukoutsaki-Monnier, Sandra Meza, Béatrice Amerein-Soltner, Rodrigue Galani et Pierre-Alain Muller

\section{(2) OpenEdition}

12 Journals

Édition électronique

URL : http://journals.openedition.org/communicationorganisation/4190

DOI : 10.4000/communicationorganisation.4190

ISSN : $1775-3546$

Éditeur

Presses universitaires de Bordeaux

Édition imprimée

Date de publication : 1 juin 2013

Pagination : 151-164

ISBN : 978-2-86781-883-7

ISSN : 1168-5549

Référence électronique

Angeliki Koukoutsaki-Monnier, Sandra Meza, Béatrice Amerein-Soltner, Rodrigue Galani et Pierre-Alain Muller, «Étudier les usages des plateformes pédagogiques numériques : quelles passerelles entre STIC et SHS ? ", Communication et organisation [En ligne], 43 | 2013, mis en ligne le 01 juin 2015 consulté le 20 avril 2019. URL : http://journals.openedition.org/communicationorganisation/4190 DOI : 10.4000/communicationorganisation.4190 


\title{
Étudier les usages des plateformes pédagogiques numériques : quelles passerelles entre STIC et SHS?
}

\author{
Angeliki Koukoutsaki-Monnier', Sondra Meza², Béatrice \\ Amerein-Soltner ${ }^{3}$, Rodrigue Golani ${ }^{4}$, Pierre-Alain Muller ${ }^{5}$
}

La question des usages ${ }^{6}$ se trouve aujourd'hui au cœur de la pédagogie numérique. Elle reflète la volonté croissante des concepteurs des supports multimédia de mieux comprendre les comportements des apprenants, mais aussi de capter et de rediriger leur attention vers des fonctionnalités ou des parcours spécifiques. L'expansion des moteurs et des systèmes de recommandation dans le secteur du marketing numérique accompagne ce mouvement. Les enquêtes menées par l'Observatoire des usages du numérique de l'Université de Strasbourg7 fournissent des données aussi bien quantitatives que qualitatives concernant l'usage des outils pédagogiques par les populations étudiées (enseignants-étudiants). Néanmoins, en se fondant majoritairement sur la parole des enquêtés, elles reproduisent davantage les représentations que ces derniers entretiennent au regard de l'objet étudié. Sans nier la valeur heuristique de ces données, qui permettent de comprendre les facteurs qui motivent ou qui freinent l'utilisation des plateformes numériques, il faut aussi admettre que celles-ci ne favorisent pas l'observation directe du comportement de l'usager. Ce dernier est davantage exploré lors d'approches développées en Sciences des technologies de l'information et

\footnotetext{
1 Angeliki Koukoutsaki-Monnier est MCF en SIC à l'Université de Haute-Alsace et membre du Centre de Recherche sur les Médiations ; angeliki.monnier@uha.fr

2 Sandra Meza est Doctorante en SDE à l'Université de Strasbourg et membre du Laboratoire Interuniversitaire des Sciences de l'Éducation et de la Communication, Professeur à l'Université du Chili ; meza@unistra.fr

3 Béatrice Amerein-Soltner est MCF en Informatique, chargée de mission "Innovations Pédagogiques Numériques » à l'Université de Haute-Alsace ; beatrice.amerein-soltner@uha.fr

4 Rodrigue Galani est responsable de l'Observatoire des Usages du Numérique, Direction des Usages du Numérique, Université de Strasbourg ; galani@unistra.fr

5 Pierre-Alain Muller est PU en Informatique à l'Université de Haute-Alsace et membre du Laboratoire de Mathématiques, Informatique et Applications ; pierre-alain.muller@uha.fr

6 L'usage chez Serge Proulx (2001, p. 139-145, p. 57) est vu sous trois angles : la « traduction » qui représente l'évaluation conceptuelle du terme, l'approche cognitive qui concerne la capacité des usagers, et l'approche sociopolitique portant sur le développement de l'usage dans la société.

$7 \mathrm{http} / / /$ services-numeriques.unistra.fr/culture-numerique/observatoire-des-usages-du-numerique.html
} 
de la communication (STIC) ainsi qu'en Sciences de l'éducation (Dessus et Marquet, 2009 ; Marquet et Coulibaly, 2007 ; Depover, Giardina et Marton, 1998 ; Caterino, Chibout et Meza, 2011). Dans cet article, nous proposons de revisiter quelques-unes de ces dernières en les mettant en parallèle avec une modélisation des usages provenant des Sciences de l'information et de la communication (SIC).

\section{Cadre théorique et hypothèses de travail}

La notion de dispositif s'encadre entre deux économies, l'une du pouvoir, inscrit dans le libéralisme où le pouvoir est sensé s'exercer sur un mode implicite et le moins coûteux possible et l'autre du savoir (Foucault, 1977/1994), dans le contexte de la société de la connaissance, construction cognitive fonctionnelle, pratique et incarnée (Linard, 1998). Le dispositif technique n'est qu'un outil limité d'apprentissage parmi d'autres, à corriger et à compléter constamment par l'accompagnement humain et social qui fonde et organise toute construction individuelle de la connaissance (Linard, 2002). Comprendre les usages des dispositifs techniques signifie les envisager en tant qu' " espaces à investir » (Hert, 1999, p. 104) et, en ce sens, questionner l'apparition - ou pas - de pratiques homogènes face à ces outils. La modélisation des comportements fournirait la possibilité de dégager des schémas génériques (patterns) qui traduiraient les lieux communs qui traversent ces pratiques ${ }^{8}$. Hormis la visée positiviste qui peut sous-tendre une telle démarche, notamment pour les concepteurs des outils numériques, interroger les usages relève aussi d'une volonté et d'un besoin sur le plan scientifique de comprendre un habitus (Bourdieu, 1980) aujourd'hui banalisé au sein des sociétés dites "développées ", et qui, à l'instar de ce que signalait Michel de Certeau (1980/1990) pour les pratiques du quotidien, reste souvent muet mais tout aussi opérant. Cela permettrait d'appréhender ce dernier sur deux niveaux : en termes de son évolution diachronique, ainsi qu'au regard de la manière dont il reflète ou forge des identités collectives propres à des communautés d'usagers (acheteurs, étudiants, etc.).

Explorer la question de l'étude des usages dans une démarche qui lie les STIC aux SHS présente un double intérêt. Ce dernier émane d'abord del'ontologie de l'objet de recherche lui-même. Les dispositifs techniques étant des construits sociaux, ils sont symptomatiques de tendances sociétales à un moment donné. Les comprendre en tant qu'objets de médiation triadique (Bruner, 1990;2000; Rabardel, 1995) revient à croiser leurs dimensions techniques, sémiotiques et sociales/pragmatiques (Peraya et al., 2002). Cependant, l'intérêt de cette approche relève aussi d'un besoin épistémologique. Il s'agit de décloisonner le savoir via des postures inter-disciplinaires (Charaudeau, 2010), continuistes

8 Pour un panorama des courants théoriques sur les usages en sciences humaines et sociales voir Maigret É., 2003 ; voir aussi les travaux de Dominique Caron et notamment Caron D. et al., 2009 ; Caron D., Delaunay-Téterel H., 2006. 
et cumulatives (Pumain, 2005) qui favorisent le dialogue et l'enrichissement entre méthodes en apparence étrangères 9 . Le pari que nous prenons dans cet article est le suivant. À partir du contexte d'utilisation de la plateforme pédagogique numérique Moodle de l'Université numérique en région Alsace (UNERA $)^{10}$, nous allons examiner la possibilité d'articuler les modélisations des usages telles qu'elles sont effectuées en Sciences des technologies de l'information et de la communication et en Sciences de l'éducation, avec une mise en perspective relevant des Sciences de l'information et de la communication.

Notre hypothèse est que loin d'être incompatibles, les problématiques qui traversent ces modélisations qui investiguent les usages, renvoient toutes, dans le sillage de la pensée foucaldienne (Foucault, 1977/1994), à la question du pouvoir des dispositifs techniques en tant que cadres organisateurs du comportement humain et de la construction du sens (Lévy, 1994 ; 1997 ; Peeters et Charlier, 1999 ; Meunier, 1999 ; Dieuaide, 2011). Elles font ainsi émerger la tension permanente entre " autonomie et contrôle " qui caractérise l'usage des TIC (Foucault, 1975 ; Flichy, 2004). Les plateformes pédagogiques numériques, davantage orientées vers l'accomplissement de parcours " prescrits ", constituent un terrain particulièrement pertinent pour explorer ces thèses. Le sens du pouvoir du dispositif, mis en parallèle avec celui de l'existence d'un ou plusieurs habitus des usagers, s'avère central pour ces applications, d'autant plus que la question de la pertinence des outils informatiques dans l'éducation reste pour certains suspendue (De Vries et Baillé, 2006).

Dans un premier temps, nous allons présenter deux modèles, l'un émanant des Sciences des technologies de l'information et de la communication (analyse comportementale) et l'autre des Sciences de l'éducation (analyse des parcours), tels qu'ils ont été décrits par Pierre-Alain Muller, Béatrice Amerein-Soltner, Manon Dargent, Rodrigue Galani et Sandra Meza (2012). Ces modèles fournissent une multitude de pistes pour observer et comprendre les usages : fréquence de visites d'un site ou d'une page web, conformité à un parcours prescrit, actions effectuées selon le profil des usagers, longueur des visites, etc. Néanmoins, chaque modèle focalise majoritairement sur un aspect particulier de la conduite de l'utilisateur. Ainsi, l'analyse comportementale, hormis les éléments qualitatifs concernant les comportements des usagers, donne davantage d'informations sur l'intensité des actions accomplies sur une plateforme numérique. En revanche, l'analyse des parcours, sans ignorer la question des fréquences, est notamment orientée vers les trajectoires des usagers. La combinaison de ces deux variantes permettrait de construire un

9 D'autres expérimentations, par exemple dans le domaine de l'e-publicité, relèvent des démarches similaires. Voir à titre indicatif Courbet D. et al., 2008.

10 https://www.e-formation.uha.fr/moodle/ 
modèle plus global pour comprendre les comportements des utilisateurs face aux outils pédagogiques numériques.

\section{Focaliser sur les intensités : l'analyse comportementale en STIC}

L'analyse comportementale déployée dans cette partie de l'article est basée sur l'observation des mouvements de souris des usagers. Le principe est de relever la position du pointeur toutes les cinq minutes, puis de dater cette position et la rapporter au contenu survolé par le pointeur. Cette technique (dite de mouse-tracking) s'inspire des techniques de suivi du regard (gaze-tracking), en postulant que la position de la souris et la position du regard présentent des points de recouvrement (par exemple lors des clics, du parcours des menus, de la lecture d'un texte, etc.), et qu'il est dès lors possible d'inférer les centres d'intérêts des usagers en se référant à l'observation de leurs mouvements de souris.

La technologie utilisée est celle développée par le laboratoire Modélisation, Intelligence, Processus et Systèmes (MIPS) de l'Université de Haute-Alsace (UHA), avec l'aide du fond de maturation Conectus (aujourd'hui la SATT Conectus). Elle inclut des outils d'analyse comportementale ainsi qu'un moteur de ciblage comportemental. Cet outil donne des indications générales sur la fréquentation du site, la durée de navigation et sur le profil des visiteurs. Il permet en outre d'ajouter de nouveaux profils; par exemple afin de distinguer les visites des étudiants de celles du personnel. Le tableau 1 propose quelques exemples d'informations relevées sur le site de la plateforme pédagogique Moodle de l'UHA.

\begin{tabular}{|l|c|c|c|}
\hline & Tous les visiteurs & Nouveaux visiteurs & Visiteurs fréquents \\
\hline Total de visites du site & 38055 & 9404 & 28727 \\
\hline Nombre de visites actives & 33493 & 8577 & 24987 \\
\hline Profondeur de visite & 8 pages/visite & 8 pages/visite & 8 pages/visite \\
\hline Temps moyen d'activité sur le site & 170 s/visite & 182 s/visite & 165 s/visite \\
\hline Temps moyen de visite du site & 394 s/visite & 431 s/visite & 381 s/visite \\
\hline Nombre de pages vues & 288471 & 74407 & 214500 \\
\hline Total de visualisation de la page & 43409 & 12531 & 30956 \\
\hline Nombre de visualisations actives & 40858 & 12036 & 28898 \\
\hline Temps moyen d'activité sur la page & $12 \mathrm{~s} /$ vue & $13 \mathrm{~s} /$ vue & 11 s/vue \\
\hline $\begin{array}{l}\text { Durée d'affichage de la page } \\
\text { Nombre de fois où la page a été page } \\
\text { d'entrée }\end{array}$ & $28 \mathrm{~s} /$ vue & $31 \mathrm{~s} /$ vue & 27 s/vue \\
\hline $\begin{array}{l}\text { Nombre de fois où la page a été page } \\
\text { de sortie }\end{array}$ & 6998 & 7302 & 17767 \\
\hline
\end{tabular}

Tableau 1 : chiffres clés sur la page d'accueil de la plateforme Moodle

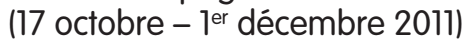


Des restitutions graphiques sont utilisées sous forme de cartes de chaleur. Les marques d'intérêts (par exemple les clics) y sont représentées sur une échelle de couleur allant du bleu au rouge. Par exemple, la carte de chaleur suivante (fig. 1) montre les centres d'intérêts des visiteurs de la page d'accueil de Moodle. L'accès aux cours génère le plus d'intérêt, suivi de près par le retour sur le portail e-services.

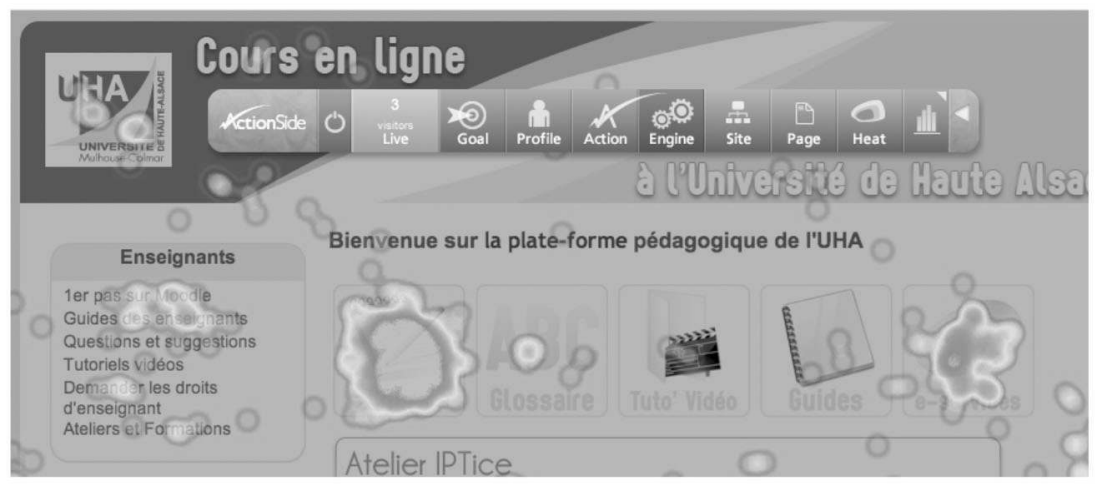

Figure 1 : carte de chaleur de la page d'accueil de Moodle. Les usagers s'intéressent principalement aux cours et au portail e-services

Une autre carte de chaleur (fig. 2) représente les zones visibles de la page d'accueil de Moodle (on parle également de profondeur de scroll). Ce type d'illustration est très indiqué pour comprendre comment les usagers s'intéressent au contenu d'une page. Car les pages fortement visitées ne sont pas forcément celles qui sont complètement visitées. Dans cet exemple, il est intéressant d'observer que les catégories de cours, classées par UFR, ne sont pas visualisées de manière homogène. On peut dans ce cadre s'interroger sur la pertinence de ce classement, ou tout du moins remettre en cause le placement de cette zone sur la page d'accueil.

Il paraît ainsi que l'observation non intrusive des actions (ou de l'absence d'action) de l'apprenant permet d'investiguer un certain nombre de questions. L'apprenant suit-il le parcours « prévu »? Exploite-t-il toutes les ressources mises à disposition ? Comment les usages varientils en fonction des profils des étudiants, profils

Figure 2 : profondeur de scroll sur la page d'accueil de Moodle. Les catégories de cours classées par UFR

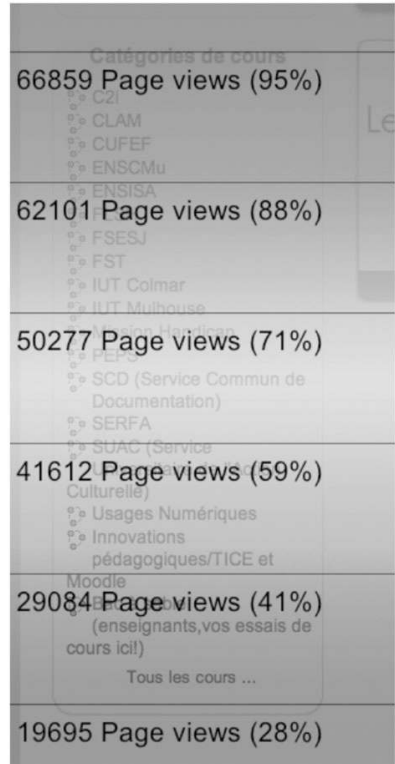
ne sont pas visibles par tous les visiteurs 
pouvant être définis en appui sur l'annuaire de l'établissement ? En outre, les fonctionnalités de l'outil ne se limitent pas à l'analyse de sites Web.

La solution offre également la possibilité de mettre en place des actions sur les pages Web afin d'influer sur le comportement de navigation d'un visiteur ; ceci pour tester par exemple différentes manières de mettre en avant un contenu peu visualisé ou pour amener le visiteur à aller sur une page spécifique.

\section{Focaliser sur les trajectoires : l'analyse des parcours en Sciences de l'Education}

Cette approche s'inscrit dans la pragmatique linguistique couplée aux outils de visualisation graphiques. Elle s'appuie sur plusieurs fondements théoriques : celui de la cartographie sémantique, définie par Christophe Tricot et al. (2006) comme un espace d'organisation d'informations et devenue aujourd'hui une activité essentielle dans le domaine de la gestion de la connaissance ; celui de l'environnement virtuel, désigné en tant que synthèse authentique permettant de repérer physiquement le déplacement ; celui de la notion d'hypertexte, proposée par Theodor Holm Nelson (1965) ${ }^{11}$ pour désigner une écriture non-séquentielle, et plus encore sa base de navigation, l'hyperlien; celui de la représentation définie par Jean-François Le Ny (1986) comme un ensemble d'informations structurées, stockées en principe dans la mémoire sémantique, que ce soit des perceptions, des significations de mots, des idées ou des concepts; enfin, celui de graphe, provenant des mathématiques, qui permet de structurer les informations sous la forme de diagrammes de correspondances abstraites et de réseaux.

L'analyse de parcours fait appel à deux types de définitions, techniques et pédagogiques. Les définitions techniques concernent les notions de nœud, lien et graphe. Un nœud est égal à un module de fonctionnalités telles qu'un forum, un éditeur de texte et un quiz. Les liens permettent de distinguer la relation et l'orientation entre les modules. La réunion de ces nœuds et liens donne forme à un graphe qui permet de représenter les parcours réels sur la base des parcours d'experts. Les définitions pédagogiques permettent de distinguer les types de tâches prescrites des types de tâches réalisées. La tâche prescrite concerne la déclaration d'objectifs dans une temporalité donnée ; la tâche réalisée, l'exécution de ces objectifs. Cet ensemble s'avère essentiel pour la description de la grammaire d'un site web à fins éducatives. Il permet de visualiser tous les chemins possibles entre un usager et l'accomplissement de la tâche prescrite dans le cadre d'un cursus universitaire ; un travail particulièrement productif qui a permis de comparer les parcours novices entre eux et avec ceux des experts ${ }^{12}$.

11 Ce concept differe de l'hypermédia et du multimédia avec lesquels il est fréquemment confondu. Lintérêt de l'hypertexte est sa possibilité d'amplifier les informations presque illimitées et de créer des lectures multiples. 12 Un autre point important à signaler est le travail en cours sur l'identification des signes composants de la sémiologie des parcours. L'état actuel de cette partie de la recherche consiste à cartographier des trajets par rapport aux clics prospectifs, rétrospectifs et d'insistance sur les modules. Mais les recherches sont encore loin d'une description cohérente de la syntaxe d'un site. 
L'un des produits de cette recherche est l'outil de traduction à langage GDF (Generic Data Framework) des données de connexion pris sur la plateforme Moodle, qui permet d'afficher autant de registres que l'on souhaite comparer. Afin d'obtenir une représentation des parcours, ce convertisseur fonctionne couplé au logiciel Gephi ${ }^{13}$, outil de visualisation de graphes. Pour une vue totale du modèle voir figure 3.

\section{Synthesis of the model of trace analysis}

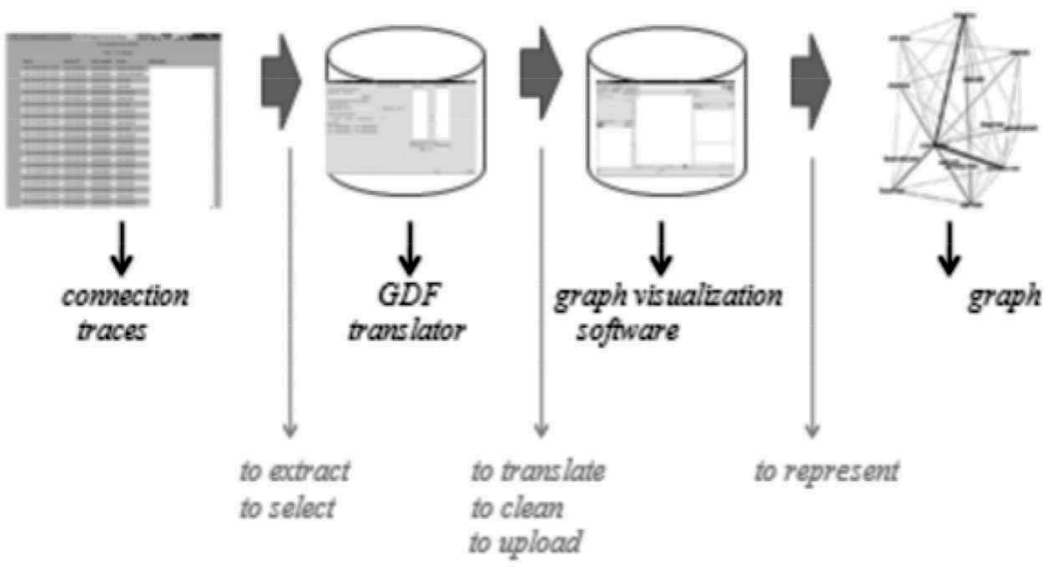

Figure 3 : principe général de l'outil de modélisation par analyse de parcours

Cette méthodologie permet de construire le graphe de parcours totaux, ainsi que les graphes moyens d'un groupe d'étudiants sélectionnés selon leur mode d'apprentissage et celui des modules utilisés. Plus particulièrement, le convertisseur de graphes GDF, en langage Java, se présente en trois parties. Un premier module se consacre à la lecture de plusieurs fichiers de données pour les stocker. Un deuxième module se sert des données pour les analyser. Il permet de reconstruire les parcours utilisateurs et d'extraire les sessions selon un filtre (par dates, par tâches). Ces analyses débouchent sur des statistiques qui servent à informer sur la fréquence d'utilisation d'un module entre un instant t et un autre, et dans la globalité du parcours. Un troisième module génère les graphes correspondants aux analyses effectuées. Il permet une meilleure visualisation des navigations sur la base des fonctionnalités statistiques. Cette visualisation en forme de graphe devrait permettre d'analyser les différents comportements de navigation et aider à leur interprétation.

La structure du convertisseur présente le lien entre les unités de navigation définies sur la base des composantes et propriétés des graphes de représentation du comportement sur un site Web. Ces composantes sont : 


\section{C\&O n ${ }^{\circ} 43$}

- parcours réel - parcours de référence (expert)

- parcours réel - fréquence d'usage des modules d'apprentissage - distance

- tâches prescrites - tâches réalisées.

Cette approche permet de rendre visible un comportement selon le mode d'apprentissage de l'étudiant concerné. Le graphe offre la possibilité de comprendre le parcours d'un étudiant à travers une tâche précise. Dans la figure 4 la représentation des parcours de quatre étudiants identifiés par des couleurs vert, rouge, jeune et violet. Les modules importants (indispensables à l'accomplissement d'une tâche) apparaissent sous forme de nœuds et la fréquence d'utilisation de ceux-ci est représentée par la taille du nœud. La distance entre les nœuds sert à représenter le nombre de modules utilisés pour arriver au nœud important suivant ${ }^{14}$.

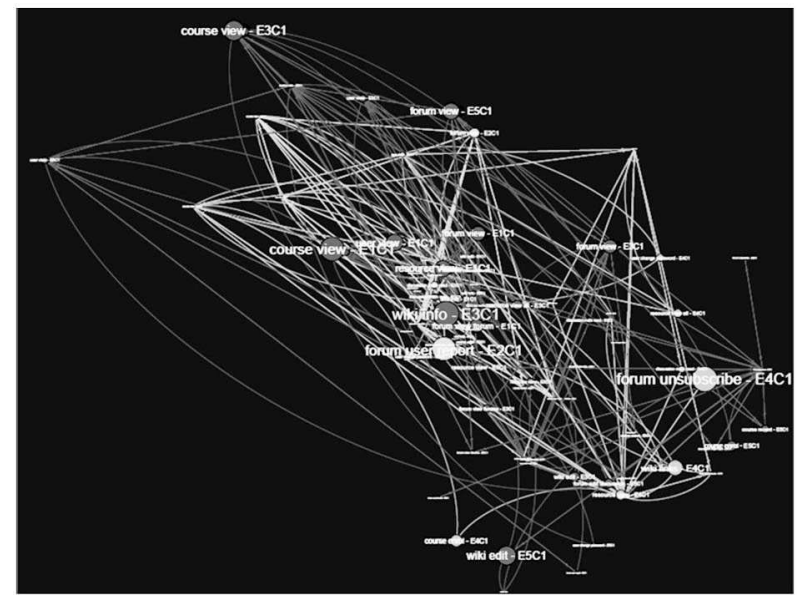

Figure 4 : visualisation des parcours au moyen de graphes

\section{Interroger les usages au prisme de la réception des médias en SIC}

En sciences de l'information et de la communication, la diversité des usages des médias a été constatée. La théorie des Usages et gratifications (Uses and Gratifications) a déjà souligné le rôle actif des publics lors de la réception de messages médiatiques (Katz E. et al., 1973-74). Au sein des études culturelles (Cultural studies), la catégorisation de Stuart Hall (1973) entre lectures hégémoniques, négociée etoppositionnelle du signe télévisuel estemblématique $\mathrm{du}$ positionnement des publics dans les processus communicationnels. Face au codage "dominant » du message par son producteur, ces derniers soit, acceptent le sens codé dans sa totalité (lecture hégémonique), soit en refusent, certaines parties (lecture négociée), soit opèrent une rupture totale avec le sens

14 En l'état actuel, les chercheurs du laboratoire LISEC à Strasbourg, travaillent sur trois types de représentations : un graphe individualisé selon le parcours de référence, un graphe individuel selon la représentation de la fréquence d'usage des modules et la longueur des parcours, un graphe des parcours totaux. 
« prescrit » et utilisent d'autres cadres de référence pour « lire » le message (lecture oppositionnelle). Il serait bien sûr illusoire de suggérer que les publics ont dans ce processus le rôle dominant. Pour expliquer le rapport des usagers aux médias en tant que négociation sociale et identitaire, Michel de Certeau (1980/1990) parle de pratiques de «braconnage » définies par les médias eux-mêmes et leurs producteurs. Transposée dans l'étude des usages des plateformes pédagogiques numériques qui nous intéresse ici, cette approche permet d'interroger comment les individus investissent ces petits espaces d'autonomie laissés au sein de l'univers numérique et comment ils négocient leurs parcours au regard des usages " prescrits " par les concepteurs de ces outils. Pour les besoins du présent travail, on désignera ainsi la négociation individuelle de la lecture du support numérique avec le terme « décodage ».

En combinant les deux variantes de trajectoires et d'intensités décrites plus haut, il est possible de proposer une visualisation des comportements des utilisateurs des plateformes pédagogiques numériques sur deux axes (schéma 1). Le premier concerne la conformité du parcours d'un usager avec celui prescrit par le concepteur du dispositif. Nicole Pignier et Benoît Drouillat (2004, p. 81) ont montré que l'architecture de l'information mise en place lors de l'élaboration d'un outil numérique (organisation hiérarchique des rubriques, positionnement dans l'espace, etc.) garantit à la fois la facilité d'accès aux contenus ainsi que la compréhension des actions à accomplir. Dans un autre registre, Jean-Paul Fourmentraux (2005) a insisté sur le rôle de l'interface informatique en tant qu' « acteur technique disséminé ». En ce sens, lorsqu'il utilise une plateforme pédagogique numérique, l'usager est amené à suivre plus ou moins un certain cheminement, un scénario, qui peut être linéaire, modulaire ou mixte (Fournier, 2003). Selon la structuration et la configuration du dispositif, l'usager a la possibilité, partielle ou totale, de négocier cette trajectoire en proposant ses propres parcours validant ou non ceux imaginés par les concepteurs. Entre acceptation ou refus du parcours prescrit, les positions intermédiaires sont nombreuses. Le deuxième axe se réfère à l'intensité d'une ou plusieurs actions accomplie(s) sur une plateforme numérique. Il permet de visualiser en termes quantitatifs la longueur de l'usage, entre investissement lourd et investissement faible, le temps étant considéré comme un atout ou une contrainte selon le cas (Séguy, 1999, p. 27).

Dans ce cadre, quatre espaces émergent correspondant à quatre types de décodages d'une plateforme pédagogique, à savoir quatre manières d'utiliser cette dernière : respecter le parcours prescrit (scénario) avec un investissement temporel conséquent (décodage dominant fort), respecter le parcours prescrit avec un investissement temporel trivial (décodage dominant faible), ne pas respecter le parcours prescrit mais investir du temps dans la visite de la plateforme (décodage atypique fort), ne pas respecter le parcours prescrit et investir peu de temps dans la visite de la plateforme (décodage atypique faible). 


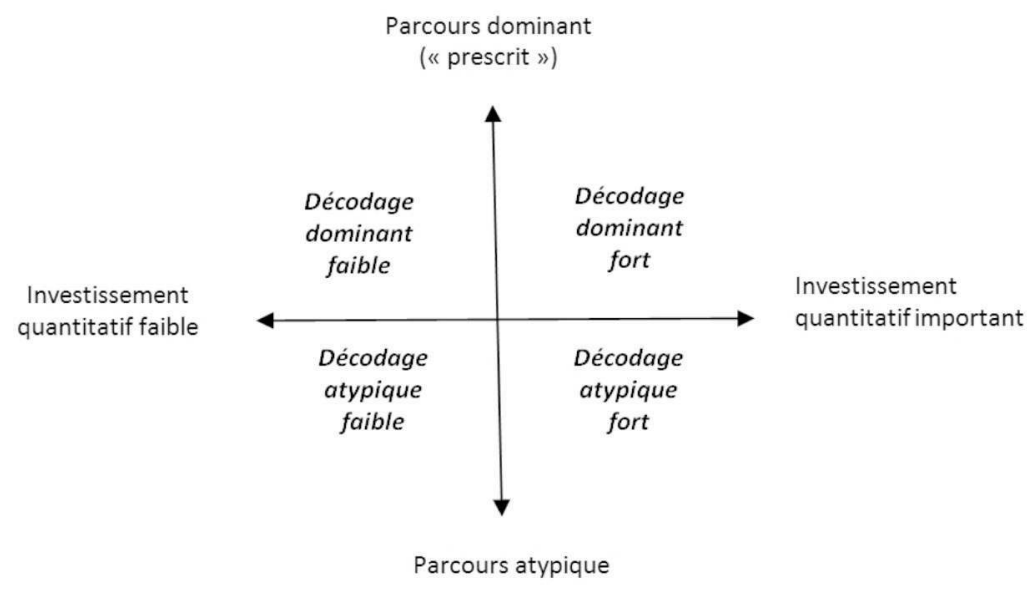

Schéma 1 : les usages selon l'analyse des "décodages "

Le schéma ci-dessus devrait être appréhendé en tant que "système de concepts, $[\ldots]$ une manière de découper a priori des phénomènes dans le réel, de les objectiver, de les classer, de leur conférer une signification » (Olivesi, 2007, p. 232). Il ressemble davantage à un signifiant dont les signifiés seraient à élaborer. Pour définir le sens de la durée par exemple, plusieurs critères seraient possibles selon les interrogations principales que les chercheurs se posent : durée d'affichage d'une page ou d'un temps d'activité, nombre de visualisations actives, nombre de pages vues, profondeur de la visite (nombre de pages par visite), etc. Ces éléments peuvent concerner à la fois une page, un module, etc. en tant qu'objet d'analyse ou bien un site dans sa totalité. Dans le même esprit, la question du parcours prescrit reste à définir car celui-ci dépend chaque fois des objectifs fixés par le concepteur et des actions qu'il souhaite voir accomplies lors d'une visite. Quant à l'échelonnage des axes et la définition des scalarités - et cela pour les deux variables étudiées -, ils se feraient sur la base de valeurs moyennes fournies par l'analyse comportementale. Enfin, dans une posture plus normative, cette modélisation permettrait d'envisager des solutions pour influer sur les conduites des usagers et de les faire passer d'une catégorie à une autre selon l'objectif fixé par le concepteur de la plateforme numérique.

Lorsqu'on se penche sur les usages de la plateforme pédagogique Moodle, par exemple, il s'avère que le parcours dominant (chemin A) qui mène à la lecture d'un cours est beaucoup moins respecté par rapport à un chemin atypique (chemin $\mathrm{B}$ ) que les usagers privilégient et qui consiste à passer par le moteur de recherche de la plateforme :

Chemin A : Page d'accueil $\rightarrow$ liste des catégories de cours $\rightarrow$ catégorie principale $\rightarrow$ sous-catégorie $\rightarrow$ cours

Chemin B : Page d'accueil $\rightarrow$ liste des catégories de cours $\rightarrow$ moteur de recherche $\rightarrow$ cours. 
Le chemin B étant plus court, il engendre inévitablement un investissement moins important de la part des utilisateurs et par conséquent une exposition plus faible aux contenus du site (tableau 2).

\begin{tabular}{|c|c|c|c|c|}
\hline & $\begin{array}{c}\text { Nombre de } \\
\text { visites }\end{array}$ & $\begin{array}{c}\text { Durée moyenne } \\
\text { de visite }\end{array}$ & $\begin{array}{c}\text { Temps moyen } \\
\text { d'activité }\end{array}$ & $\begin{array}{c}\text { Nombre de } \\
\text { pages affichées } \\
\text { en moyenne }\end{array}$ \\
\hline $\begin{array}{c}\text { Parcours } \\
\text { dominant }\end{array}$ & 506 & 638 & 324 & 13 \\
\hline $\begin{array}{c}\text { Parcours } \\
\text { atypique }\end{array}$ & 1002 & 542 & 281 & 9 \\
\hline
\end{tabular}

Tableau 2 : chiffres clés sur la page d'accueil de la plateforme Moodle selon l'analyse des décodages (14 mai - 29 mai 2012)

Sur la base de ces moyennes, il est possible de situer chaque usager individuellement par rapport à l'utilisation qu'il fait du site (chemin choisi, durée, etc.). Ces données peuvent aussi amener à s'interroger sur la structuration générale de la plateforme numérique, ainsi qu'au sens donné au « parcours dominant». La tendance des usagers à privilégier les parcours les plus rapides s'avère par exemple un élément important.

\section{Conclusion}

La mise en perspective de modèles issus des STIC et des Sciences de l'éducation, relatifs aux usages des plateformes pédagogiques numériques, avec une réflexion et un schéma émanant des SIC permet de valider l'existence d'un fond commun qui caractérise ces approches. En interrogeant le rapport des hommes et des femmes aux dispositifs techniques, celles-ci revisitent la question du pouvoir des dispositifs techniques en tant qu'objets fluides, en construction et reconfiguration perpétuelle, résultats de stratégies de pouvoir des acteurs sociaux et générateurs de cadres de signification et d'action. Elles suggèrent plus particulièrement la diversité des problématiques liées à l'étude de ces derniers, centrées notamment autour de deux axes : d'abord, celui de la nature des dispositifs techniques en tant qu'outils " limités ", à corriger et à compléter à des fins d'apprentissage (Linard, 1998 ; 2002), ou en tant qu'espaces à investir susceptibles de générer des pratiques homogénéisées (Hert, 1999); puis, celui des répercussions de l'usage des dispositifs techniques pour l'économie du pouvoir et du savoir (Foucault, 1975 ; 1977/1994), pour la structuration de dichotomies sémantiques comme celles entre symbolique/ technique et humain/non-humain (Peeters et Charlier, 1999), ainsi que pour l'organisation du comportement humain et la construction du sens de façon générale (Lévy, 1994 ; 1997).

En ce sens, les SHS peuvent prolonger les modèles issus des STIC en proposant des schémas interprétatifs des usages, des conceptualisations qui 
rendent davantage signifiants les comportements. Comme c'est le cas de toute modélisation, il s'agit de constructions simplificatrices des phénomènes complexes dont toutes les réalités ne sont pas prises en compte (motivations des usagers, émotions, statut social, etc.). Néanmoins, ce croisement d'approches ouvre des perspectives pour étudier les usages. Dans ce cadre, l'existence d'identités collectives fondées sur des pratiques communes des outils numériques s'avère particulièrement intéressante à explorer : selon les secteurs (e.g. plateformes pédagogiques versus e-commerce), selon les publics (e.g. étudiants-enseignants), etc.

\section{BIBLIOGRAPHIE}

BOURDIEU P., Le sens pratique, Paris, Éd. de Minuit, 1980.

BRUNER J. S., Acts of Meaning, Cambridge, Mass., Harvard University Press, 1990.

BRUNER J. S., Cultures et modes de pensée, L'esprit humain dans ses ceuvres, Paris, Retz, 2000, (éd. originale : Actual Minds, Possible Words, Cambridge, Mass., Harvard University Press, 1986).

CARON D., BEUSCART J.-S., PRIEUR C., PISSARD N., « Pourquoi partager mes photos de vacances avec des inconnus ? Les usages de Flickr », Réseaux, 154, 2009, p. 91-129.

CARON D., DELAUNAY-TÉTEREL H., « La production de soi comme technique relationnelle. Un essai de typologie des blogs par leurs publics », Réseaux, 138, 2006, p. 15-71.

CATERINO P., CHIBOUT K., MEZA S., " Profils de communication, profils d'apprentissage et usages sur une plateforme pédagogique en ligne : étude exploratoire ", Actes du Colloque EPAL (Échanger pour apprendre en ligne), Grenoble, 2011, p. 24-25, http://w3.u-grenoble3.fr/epal/dossier/ 03_pro/ resumes_epal.pdf

CERTEAU de M., Linvention du quotidien, 1. Arts de faire, Paris, Gallimard, 1980/1990.

CHARAUDEAU P., "Pour une interdisciplinarité "focalisée" dans les sciences humaines et sociales ", Questions de communication, 17, 2010, http://www.patrickcharaudeau.com/Pour-une-interdisciplinarite.html

COURBETD.,VANHUELE M.,LAVIGNE F., «Les effets persuasifs del'e-publicité perçue "sans conscience" en vision périphérique. Implications pour les recherches sur la réception des médias ", Questions de communication, 14, 2008, p. 197-219.

DEPOVER C., GIARDINA M., MARTON P., Les environnements d'apprentissage multimédia, Analyse et conception, Paris, L'Harmattan, 1998.

DESSUS P., MARQUET P. dir., «Les effets des dispositifs d'EAD sur l'enseignement et l'apprentissage ", Distances et Savoirs, 7, $\mathrm{n}^{\circ}$ thématique 1, Paris : Lavoisier, 2009, p. $1-129$.

DIEUAIDE P., «Travail cognitif », Communications, 88, dossier spécial « Cultures du numérique », 2011, p. 177-185. 
DE VRIES E., BAILLÉ J., « Apprentissages : référents théoriques pour les EIAH », in GRANDBASTIEN M., LABAT J.-M. dir., Environnements informatiques pour l'apprentissage humain, Paris, Hermès, 2006, p. 25-47.

FLICHY P., "L'individualisme connecté entre la technique numérique et la société », Réseaux, 124, 2004, p. 17-51.

FOUCAULT M., Surveiller et punir,Paris, Gallimard, 1975.

FOUCAULT M., Dits et écrits, T. II, Paris, Gallimard, 1977/1994.

FOURMENTRAUX J.-P., « Programmer l'interface. Les ambivalences d'une matrice relationnelle », Questions de communication, 8, 2005, p. 245-259.

FOURNIER J., Scénarisation et multimédia. Processus de scénarisation interactive, Québec, Les Presses de l'Université Laval, 2003.

HALL S., Encoding and Decoding in the Television Discourse, Birmingham, Centre for Contemporary Cultural Studies, 1973.

HERT P., «Internet comme dispositif hétérotopique », Hermès, 25, 1999, p. 93-107.

KATZ E., BLUMLER J. G., GUREVITCH M., «Uses and Gratifications Research », The Public Opinion Quarterly, 37, 1973-1974, p. 509-23.

LE NY J.-F., « Les représentations (vues sous l'angle psychologique) », École d'été «Approche des sciences cognitives : les représentations », Bonas, 30 juin-11 juillet 1986.

LÉVY P., L'intelligence collective. Pour une anthropologie du cyberespace, Paris, Éd. La Découverte, 1994.

LÉVY P., La cyberculture, Paris, Éd. Odile Jacob, 1997.

LINARD M., "L'écran de TIC, “dispositif” d'interaction et d'apprentissage : la conception des interfaces à la lumière des théories de l'action ». Colloque dispositifs et médiations des savoirs, Université Catholique de Louvain, 1998, http://edutice.archivesouvertes.fr/edutice-00000913/fr/

LINARD M., " Conception de dispositifs et changement de paradigme en formation », Éducation Permanente, 152, 2002, p. 143-155.

MAIGRET É., Sociologie de la communication et des médias, Paris, Armand Colin, 2003.

MARQUET P., COULIBALY B., " Le concept de conflit instrumental : une hypothèse explicative des non-usages des TIC », Dossiers d'ingénierie éducative, hors série «TICE, l'usage en travaux », 2007, p. 61-69.

MEUNIER J.-P., « Dispositif et théories de communication », Hermès, 25, 1999, p. 83-91.

MULLER P.-A., AMEREIN-SOLTNER B., DARGENT M., GALANI R., MEZA S., "Vers une meilleure connaissance des usages dans les environnements de travail numériques et les plateformes pédagogiques ", Colloque International de l'Université à l'Ėre du Numérique (CIUEN), Lyon, 2012.

NELSON T. H., "Complex information processing : a file structure for the complex, the changing and the indeterminate ", Association for Computing Machinery: Proceedings of the 1965 20th National Conference, New York, ACM Press, 1965, p. 84-100.

OLIVESI S., " Le travail du concept. Théories, modèles, catégories », OLIVESI S. dir., Introduction à la recherche en SIC, Grenoble, Presses Universitaires de Grenoble, 2007, p. $220-240$. 
PEETERS H. CHARLIER P., « Contributions à une théorie du dispositif », Hermès, 25, 1999, p. 15-23.

PERAYA D., VIENS J., KARSENTI T., « Formation des enseignants à l'intégration pédagogique des TIC : Esquisse historique des fondements, des recherches et des pratiques ", Revue des sciences de l'éducation, 28(2), 2002, p. 243-264.

PIGNIER N., DROUILLAT B., Penser le webdesign. Modèles sémiotiques pour les projets multimédias, Paris, L'Harmattan, 2004.

PROULX S., «Usages de l'Internet : la "pensée-réseaux” et l'appropriation d'une culture numérique ", GUICHARD É. dir., Comprendre les usages de l'Internet, Paris, Éditions Rue d'Ulm, Presses de l'École Normale Supérieure, 2001, p. 139-145.

PUMAIN D., "Cumulativité des connaissances », Revue européenne des sciences sociales, XLIII-131, 2005, http://ress.revues.org/357

RABARDEL P., Les hommes et les technologies, Approche cognitive des instruments contemporains, Paris, Armand Colin, 1995.

SÉGUY F., Les produits interactifs et multimédias, Grenoble, Presses universitaires de Grenoble, 1999.

TRICOT C., ROCHE C., FOVEAU C.-E., REGUIGUI S., « Cartographie sémantique de fonds numériques scientifiques et techniques ", Document numérique, 9(2), 2006, p. 12-35, www.cairn.info/revue-document-numerique-2006-2-page-12.htm

Résumé : Dans cet article, nous proposons de revisiter deux études relatives aux usages de plateformes pédagogiques numériques, telles qu'elles ont été développées en Sciences des technologies de l'information et de la communication (STIC) et en Sciences de l'éducation, en les mettant en parallèle avec un schéma conçu en Sciences de l'information et de la communication (SIC). Loin d'être incompatibles, les problématiques qui traversent ces différentes approches interrogent la question du pouvoir des dispositifs techniques en tant que cadres organisateurs du comportement humain et de la construction du sens. Le sens du pouvoir du dispositif, mis en parallèle avec celui de l'existence d'un ou plusieurs habitus des usagers, s'avère central pour ces analyses.

Mots-clés : Usages, plateformes pédagogiques numériques, modélisations, dispositifs, passerelles entre STIC et SHS.

Abstract : In this paper, we first reconsider two models that analyse the usages of educational digital platforms, as developed by researchers in Sciences of Information and Communication Technologies and Sciences of Education. The latter are then used as a background to a schema conceived in Information and Communication Sciences. Far from being incompatible, these approaches seem to question the power of technical devices as organising frames constructing behaviours and meanings. The existence of specific habitus imputed to particular usages reveals to be central in these studies.

Keywords : Usages, Educational digital platforms, Modelling, Technical devices, Links between Technology Sciences and Human Sciences 